\title{
Propagation of Surface Plasmon Polariton in the Single Interface of Gallium Lanthanum Sulfide and Silver
}

\author{
Rakibul Hasan SAGOR, Md. Ghulam SABER*, and Md. Ruhul AMIN \\ Department of Electrical and Electronic Engineering, Islamic University of Technology, Board Bazar, Gazipur-1704, \\ Bangladesh \\ *Corresponding author: Md. Ghulam SABER E-mail: saber.iut@gmail.com
}

\begin{abstract}
The propagation characteristics of the surface-plasmon-polariton (SPP) mode in the single interface of silver (Ag) and gallium lanthanum sulfide (GLS) have been studied both analytically and numerically. The obtained numerical results show an excellent agreement with the analytical ones. The locations of the spatial resonance point along the direction of propagation were determined for the dielectric and the metal.
\end{abstract}

Keywords: Surface-plasmon-polariton, gallium lanthanum sulfide, glass-metal interface

Citation: Rakibul Hasan SAGOR, Md. Ghulam SABER, and Md. Ruhul AMIN, "Propagation of Surface Plasmon Polariton in the Single Interface of Gallium Lanthanum Sulfide and Silver," Photonic Sensors, 2014, 4(1): 58-62.

\section{Introduction}

In the past few years, research interest in the field of plasmonics has increased significantly due to the potential application in sensing, communications, computing, and imaging [1-3]. The enhanced propagation of the surface-plasmonpolariton (SPP) can be a pioneering step towards the miniaturization of integrated photonic devices. Recently, chalcogenide glasses have drawn more attention of the researchers because of their photosensitivity to the visible light and transparent behavior in the mid-infrared region of the electromagnetic spectrum [4]. They provide strong confinement and dispersion which enhance the SPP propagation distance as well as the signal strength.

R. Ahmad et al. [5] demonstrated an optical parametric oscillator (OPO) based on the chalcogenide glass that holds promise for realizing mid-infrared OPOs employing currently available optical sources in the optical communication wavelength region. T. North et al. [6] reported the fabrication and characterization of a pulsed fiber ring laser based on the chalcogenide glass. A. Al-kadry et al. [7] reported the generation of a broadband supercontinuum in chalcogenide wires by avoiding the two-photon absorption effects. P. Kumar Maharana et al. [8] proposed a method for the electric field enhancement in the surface plasmon resonance bimetallic configuration based on the chalcogenide prism.

Herein, we provide a detailed investigation into SPP propagation characteristics in the single interface of $\mathrm{Ag}$ and gallium lanthanum sulfide (GLS). The properties we have studied are the SPP wavelength, penetration depth into both Ag and GLS layers, and electric field strength at different distances from the interface for different wavelengths of the input signal. Numerically obtained results have been compared with the

Received: 24 August 2013/ Revised version: 13 September 2013

C The Author(s) 2013. This article is published with open access at Springerlink.com

DOI: $10.1007 / \mathrm{s} 13320-013-0141-4$

Article type: Regular 
analytical results, and an excellent agreement has been observed. To the best of our knowledge, this is the first time one has analyzed the propagation behavior of the SPP mode in the single interface of GLS and Ag.

\section{Material models}

The frequency dependent permittivity function of the second order single pole-pair Lorentz model is given by

$$
\varepsilon_{r}(\omega)=\varepsilon_{\infty}+\frac{\omega_{o}^{2}\left(\varepsilon_{s}-\varepsilon_{\infty}\right)}{\omega_{o}^{2}+j 2 \delta \omega-\omega^{2}}
$$

where $\varepsilon_{\infty}$ is the infinite frequency relative permittivity, $\varepsilon_{s}$ is the zero frequency relative permittivity, $j$ is the imaginary unit, $\delta$ is the damping co-efficient, and $\omega_{o}$ is the frequency of the pole pair.

The frequency dependent permittivity function of the Lorentz-Drude 6 (six) pole model is given by

$$
\varepsilon_{r}(\omega)=1-\frac{f_{o} \omega_{p}^{2}}{\omega^{2}-j \Gamma_{o} \omega}+\sum_{i=1}^{5} \frac{f_{i} \omega_{p}^{2}}{\omega_{o i}^{2}+j \Gamma_{i} \omega-\omega^{2}}
$$

where $\omega_{p}$ is the plasma frequency, $\Gamma_{i}$ is the damping frequency, $f_{i}$ is the oscillator strength, $j$ is the imaginary unit, and $\omega_{o i}$ is the resonant frequency.

The second order single pole-pair Lorenz model has been used to model GLS, and the 6-pole Lorentz-Drude model has been used to model Ag. The modeling parameters for the materials have been obtained from different resources. For Ag, we used the parameters obtained by Rakic et al. [9], and for GLS, we used the parameters determined by R. H. Sagor [10].

\section{Methodology of analysis}

A two-dimensional simulation model has been developed based on the finite-difference time-domain (FDTD) [11] algorithm in order to simulate the glass-metal structure. The original FDTD algorithm formulated by Yee does not account for the frequency dependent permittivity of the materials. Therefore, we used the auxiliary differential equation (ADE) based general FDTD algorithm in order to incorporate the frequency dependent dispersion property of the materials. This algorithm can handle the case when there are materials with different dispersion properties. The perfectly matched layer [12] is also used in order to avoid reflection of incident waves from the boundaries.

Considering the material dispersion, the frequency-dependent electric flux density can be given as

$$
D(\omega)=\varepsilon_{o} \varepsilon_{\infty} E(\omega)+P(\omega) .
$$

The general Lorentz model is given by

$$
P(\omega)=\frac{a}{b+j c \omega-d \omega^{2}} E(\omega)
$$

which can be written in time-domain through the inverse Fourier transform as

$$
b P(t)+c P^{\prime}(t)+d P^{\prime \prime}(t)=a E(t) .
$$

The FDTD solution for the first order polarization of (5) can be expressed as

$$
P^{n+1}=C_{1} P^{n}+C_{2} P^{n-1}+C_{3} E^{n}
$$

where

$$
C_{1}=\frac{4 d-2 b \Delta t^{2}}{2 d+c \Delta t}, C_{2}=\frac{-2 d-c \Delta t}{2 d+c \Delta t}, C_{3}=\frac{2 a \Delta t^{2}}{2 d+c \Delta t} .
$$

The values of $C_{1}, C_{2}$, and $C_{3}$ depend on the material under consideration.

Finally, the electric field intensity becomes

$$
E^{n+1}=\frac{D^{n+1}-\sum_{i}^{N} P^{n+1}}{\varepsilon_{o} \varepsilon_{\infty}}
$$

where $D^{n+1}$ is the update value of the electric flux density calculated using the FDTD algorithm.

\section{Specification of the structure}

The single interface glass-metal structure that was used for simulation is given in Fig. 1. The width of the Ag layer is taken as $500 \mathrm{~nm}$, and a $500-\mathrm{nm}$ cladding of the GLS glass is given on the top of Ag.

The step size is taken as $\Delta x=2 \mathrm{~nm}, \Delta y=2 \mathrm{~nm}$, and the time step has been set as

$$
\Delta t=0.95 / c \sqrt{\frac{1}{\Delta x^{2}}+\frac{1}{\Delta y^{2}}}
$$


where $c$ is the speed of light and taken as $c=3 \times 10^{8} \mathrm{~ms}^{-1}$. We found it sufficient to satisfy the Courant stability condition [13].

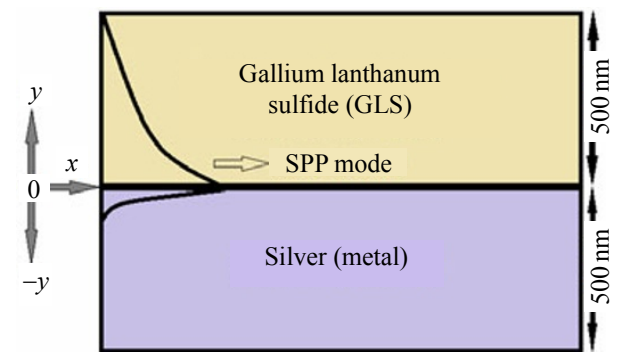

Fig. 1 Structure of the single interface used for simulation.

\section{Results and discussion}

The SPP wavelength is given by

$$
\lambda_{\mathrm{SPP}}=\lambda_{o} \sqrt{\left(\varepsilon_{d}+\varepsilon_{m}^{\prime}\right) / \varepsilon_{d} \varepsilon_{m}^{\prime}}
$$

where $\lambda_{o}$ is the free space wavelength, $\varepsilon_{d}$ is the real part of the complex relative permittivity of the dielectric, and $\varepsilon_{m}^{\prime}$ is the real part of the complex relative permittivity of the metal. In Fig. 2, the plot of the normalized SPP wavelength obtained by using both equation and simulation has been given, and we can observe a very good agreement between both the results.

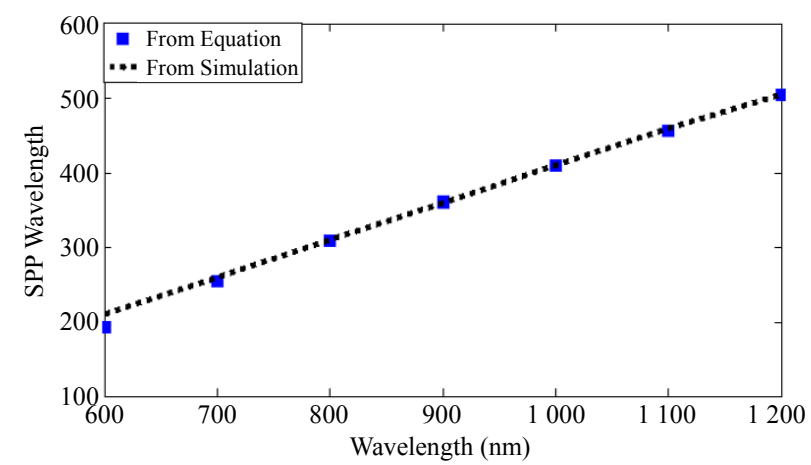

Fig. 2 SPP wavelength calculated analytically and numerically.

The equation for the penetration depth into the dielectric and metal are given by

$$
\begin{aligned}
& \delta_{d}=1 / k_{o}\left|\left(\varepsilon_{d}+\varepsilon_{m}^{\prime}\right) / \varepsilon_{d}{ }^{2}\right|^{0.5} \\
& \delta_{m}=1 / k_{o}\left|\left(\varepsilon_{d}+\varepsilon_{m}^{\prime}\right) / \varepsilon_{m}{ }^{2}\right|^{0.5}
\end{aligned}
$$

where $k_{o}=2 \pi / \lambda_{o}$ is the wave-vector for light in the free space. The plot of the penetration depth into silver and GLS layers obtained both analytically and numerically is given in Fig. 3 which shows a quite good agreement.

The penetration depth in GLS is higher than that in the metallic layer. This happens because the SPP mode field attenuates more in the metal, and the dissipated energy is transformed into heat.

The minor disparity between the numerical and analytical results in Figs. 2 and 3 can be attributed to the difficulty in determining the exact location where the SPP gets matured. Also, in the simulation model, the material defining parameters are not $100 \%$ accurate.

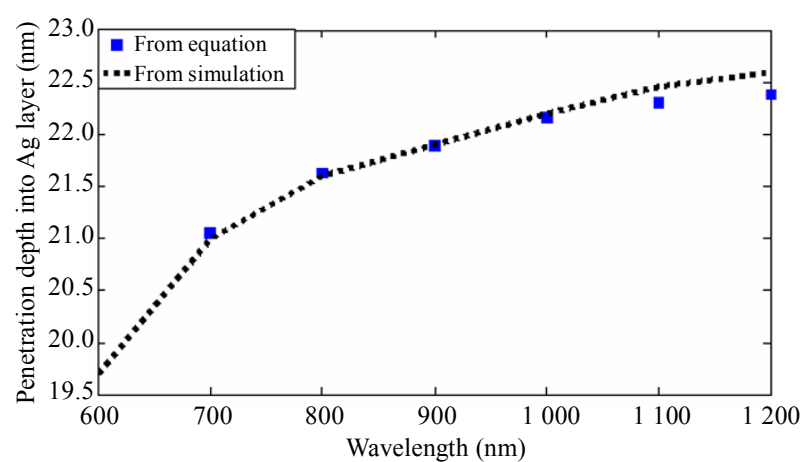

(a)

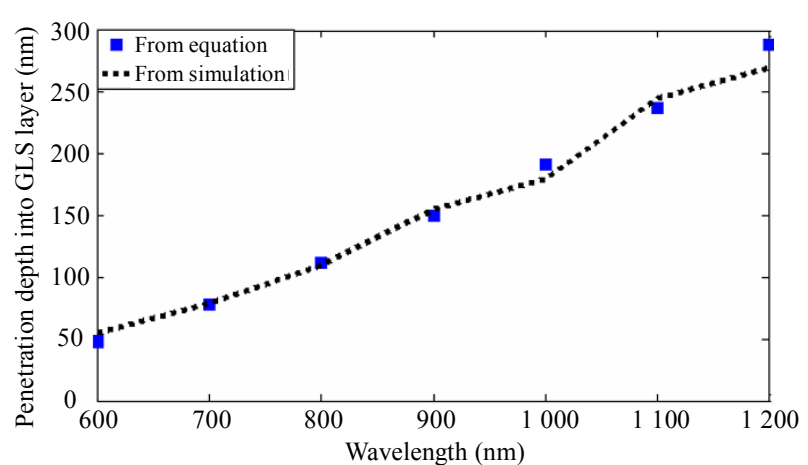

(b) layer.

Fig. 3 Field penetration depth into (a) Ag layer and (b) GLS

The variations of the electric field strength in the $y$-direction in both silver and GLS are presented in Fig. 4. For Ag, we recorded the electric field from $5 \mathrm{~nm}$ to $30 \mathrm{~nm}$ in the step of $5 \mathrm{~nm}$ in the $y$-direction, and for GLS, we recorded from $10 \mathrm{~nm}$ to $60 \mathrm{~nm}$ in the step of $10 \mathrm{~nm}$ for different input signal wavelengths. It can be observed that the electric field strength decreases as we go away from the interface, and the amount of decrease is more in the case of Ag. This is the reason why the SPP mode 
field decreases exponentially in strength as one goes further from the interface in the $y$-direction. This happens due to the skin effect of the SPP mode in the Ag and GLS layers. The resonance mode is also clearly visible in Fig. 4 with peak amplitudes centered at the input signal wavelength of $525 \mathrm{~nm}$.

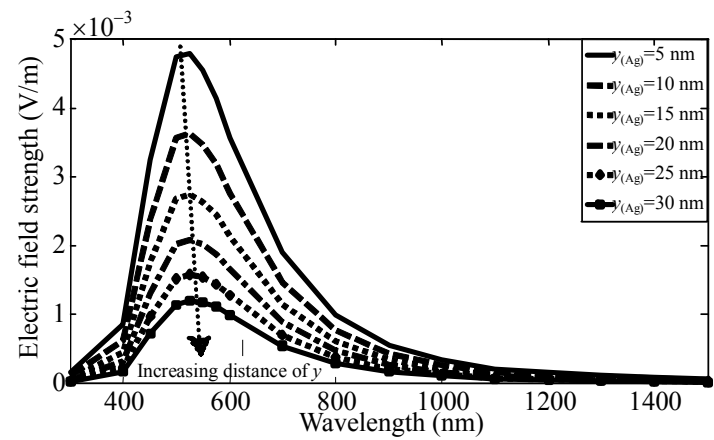

(a)

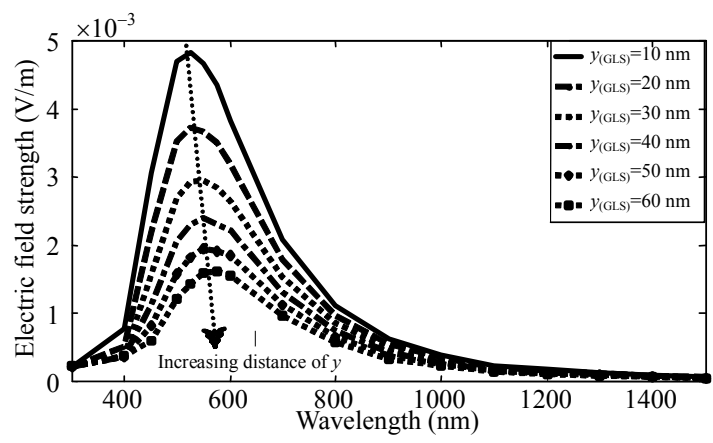

(b)

Fig. 4 Electric field strength as a function of the distance (a) in Ag layer (b) in GLS layer.

The electric field distribution in the Ag-GLS interface has been given in Fig. 5 .

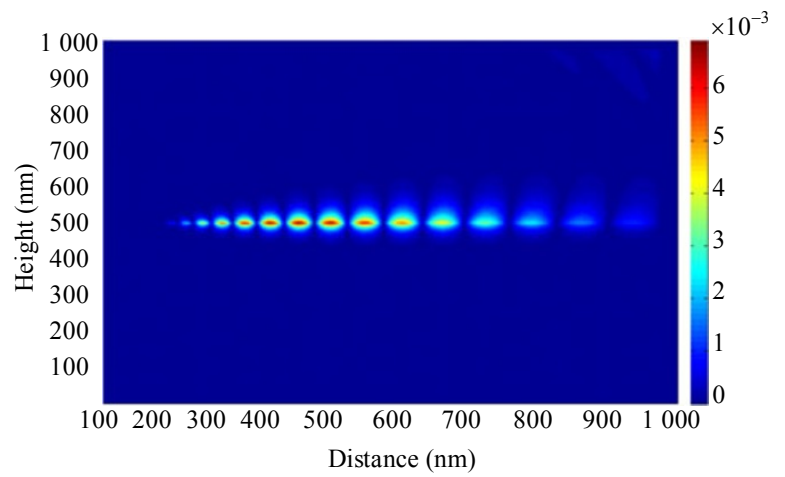

Fig. 5 Electric field distribution in the single interface of $\mathrm{Ag}$ and GLS.

\section{Conclusions}

The propagation characteristics of the SPP mode in the GLS-Ag interface have been presented. The SPP wavelength and penetration depth have been determined using both simulation and analytical equations, which have the good agreement. The electric field strength decreases exponentially in the $y$-direction which is also consistent with the theoretical results. This analysis will be helpful in the fabrication process of highly integrated photonic devices using GLS.

\section{Acknowledgment}

The authors would like to acknowledge the support of Islamic University of Technology.

Open Access This article is distributed under the terms of the Creative Commons Attribution License which permits any use, distribution, and reproduction in any medium, provided the original author(s) and source are credited.

\section{References}

[1] W. Saj, T. Antosiewicz, J. Pniewski, and T. Szoplik, "Energy transport in plasmon waveguides on chains of metal nanoplates," Opto-Electronics Review, 2006, 14(3): 243-251.

[2] S. A. Maier, Plasmonics: fundamentals and applications, Berlin Heideberg: Springer, 2007: 53.

[3] W. Dickson, G. A. Wurtz, P. R. Evans, R. J. Pollard, and A. V. Zayats, "Electronically controlled surface plasmon dispersion and optical transmission through metallic hole arrays using liquid crystal," Nano letters, 2008, 8(1): 281-286.

[4] M. Asobe, "Nonlinear optical properties of chalcogenide glass fibers and their application to all-optical switching," Optical Fiber Technology, 1997, 3(2): 142-148.

[5] R. Ahmad and M. Rochette, "Chalcogenide optical parametric oscillator,” Optics Express, 2012, 20(9): 10095-10099.

[6] T. North and M. Rochette, "Fabrication and characterization of a pulsed fiber ring laser based on $\mathrm{As}_{2} \mathrm{~S}_{3}$," Optics Letters, 2012, 37(4): 716-718.

[7]A. Al-kadry, C. Baker, M. El Amraoui, Y. Messaddeq, and M. Rochette, "Broadband supercontinuum generation in $\mathrm{As}_{2} \mathrm{Se}_{3}$ chalcogenide wires by avoiding the two-photon absorption effects," Optics Letters, 2013, 38(7): 1185-1187. 
[8] P. Kumar Maharana, S. Bharadwaj, and R. Jha, "Electric field enhancement in surface plasmon resonance bimetallic configuration based on chalcogenide prism," Journal of Applied Physics, 2013, 114(1): 014304-01-014304-04.

[9] A. D. Rakic, A. B. Djurišic, J. M. Elazar, and M. L. Majewski, "Optical properties of metallic films for vertical-cavity optoelectronic devices," Applied optics, 1998, 37(22): 5271-5283.

[10] R. H. Sagor, "Plasmon enhanced symmetric mode generation in metal-insulator-metal structure with Kerr nonlinear effect," International Journal of
Computer Applications, 2012, 50(18): 24-28.

[11] K. Yee, "Numerical solution of initial boundary value problems involving Maxwell's equations in isotropic media," IEEE Transactions on Antennas and Propagation, 1966, 14(3): 302-307.

[12] J. P. Berenger, "A perfectly matched layer for the absorption of electromagnetic waves," Journal of Computational Physics, 1994, 114(2): 185-200.

[13] A. Taflove and S. C. Hagness, Computational electrodynamics, Boston, London: Artech house, 2000: 133. 